\title{
EXCITATION DEPENDENT GF-VALUES AND DEPTH DEPENDENT MICROTURBULENCES
}

\author{
Toshio Hasegawa \\ Hokkaido University of Education \\ Asahikawa, 070, Japan
}

Abstract

The velocity of microturbulence is frequently determined from Fe I lines. Unfortunately classical gf-values of this element have excitation dependent errors. In the absolute curve of growth analysis of some F-type stars with new gf-values, the author found that a great part of the depth dependence (i.e. excitation dependence) of microturbulent velocity, which had been derived by many authors with old gfvalues, is the consequence of these errors.

The errors of old gf-values increased with excitation potential and the errors were compensated by adopting velocities of microturbulence decreasing with excitation potential. On the other hand, gf-values of ionized elements (e.g. Ti II and Fe II) are not changed as much as those of Fe $I$, accordingly the ionization dependence can also be eliminated.

\section{ON THE STRUCTURAL AND STOCHASTIC MOTIONS IN THE SOLAR AND STELLAR ATMOSPHERES}

\author{
E.I. Mogilevsky \\ Izmiran, USSR, Academy of Sciences \\ Moscow, U.S.S.R.
}

Abstract

Existence of discrete structures of velocity $\bar{V}$ and magnetic field $\bar{B}$ in stellar atmospheres follows from the Vlasov's integral equation

$$
\int \xi \mathrm{d} \tau=F(v \bar{B}, T \ldots)
$$

where $\mathcal{F}(u, \dot{v}, r)$ is a statistical function of the distribution of the elements of matter having masses $m=\frac{\boldsymbol{P}}{\dot{v}}$ and velocities, $v, d \tau$ is elementary phase volume, $F$ is functional which arrange the ties between macrovelocity, magnetic field, temperature, etc., providing self containment of cosmic plasma. The self containment of cosmic plasma 
leads to some specific fundamental properties of solar and stellar atmospheres.

Equation (1) has discrete solutions, implying the existence of the structural hierarchy, with definite characteristics for the density, velocity, magnetic field, etc. As a result, "strange" phenomena take place in the solar and stellar atmospheres, for example: Magnetization, non-balancing of the magnetic fluxes in $N$ and $S$ polarities, surprising fast dynamics of the active phenomena, the non-classical values of electro and conductivity, etc.

The predominance of filamentary structures, which are well observed in the solar atmosphere, leads to the stochasticity and quasiperiodicity of motions.

I U E OBSERVATIONS OF CIRCUMSTELLAR LINES AND MASS LOSS FROM B-STAR

\author{
S.P. Tarafdar, K.S. Krishna Swamy and M.S. Vardya \\ Tata Institute of Fundamental Research \\ Homi Bhabha Road, Bombay 400005 , India
}

\title{
Abstract
}

The circumstellar lines due to $A^{1} \Pi-x^{1} \Sigma$ transition of $C O$ has been identified for the first time in the spectra of an early type star. The spectrum, taken from the International Ultraviolet Explorer between 1150 - $1900 \AA$, of 9 Cep, a B2Ib star with $E(B-V)=0.47$, shows not only the interstellar absorption lines of $\infty$ but also absorption features shifted towards short wavelength relative to the interstellar lines. The amount of shifts towards short wavelength from the $1-0,2-0,3-0,4-0, \&$ 6 - o interstellar absorption bands of co has been found to be fairly close and corresponds to a velocity of $450 \mathrm{~km} \mathrm{sec}{ }^{-1}$. All attempts to identify these set of lines in a consistent way with atomic or ionic lines have yielded essentially negative results. This has led us to tentatively identify these lines due to circumstellar $\infty$ around 9 Cep. An estimate of rate of mass loss from the circumstellar co-column density leads to a value of $\dot{\mathrm{m}} \geqslant 10^{-11} \mathrm{M}_{\odot} \mathrm{yr}^{-1}$. 